proposed an alternative explanation: POA is primarily active against PanD but PanD is only essential if the bacteria are expressing a stringent response, the other genes associated with resistance in some way disrupt the stringent response and eliminate the sensitive phenotype. This suggests a critical role for the stringent response in the life cycle of $M$. tuberculosis as compounds are being developed that target this pathway we suggest these compounds are particularly promising compounds for the treatment of tuberculosis.

6.4

doi: 10.15789/2220-7619-2018-4-6.4

\section{IDENTIFICATION OF MUTATIONS OF RESISTANCE TO FLUOROQUINOLONES, AMINOGLYCOSIDES AND ETHAMBUTOL IN RIFAMPICIN-RESISTANT MYCOBACTERIUM TUBERCULOSIS}

I.V. Bakshtanovskaya, T.F. Stepanova, A.N. Ozerova, E.A. Zmatrakova, I.V. Ozhirel'eva

Tyumen Regional Infectious Pathology Research Institute, Tyumen, Russia

The aim of the study was to identify resistance mutations to second-line anti-tuberculosis drugs in patients with Mycobacterium tuberculosis clinical samples resistant to rifampicin. Samples of biomaterial from 35 adult residents of the Tyumen region in West Siberia with established by GeneXpert system presence of rifampicin-resistant mycobacteria tuberculosis (MBT) were examined in our study using the MTBDRsl kit (Hain Lifescience, Nehren, Germany) according to the manufacturer's instructions. The mutations were identified in genes encoding DNA gyrase (gryA), 16S RNA (rrs), and arabinosyltrasferase $(e m b B)$ associated with resistance to fluoroquinolones (FLQ), injectable aminoglycosides/cyclic peptides $(\mathrm{AG} / \mathrm{CH})$ and ethambutol (EMB) respectively.

In 9 of the examined samples (25.7\%) the MBT resistance to all three groups of drugs was revealed. In the remaining 26 samples, the MBT sensitivity to one or two groups of drugs can be assumed. Samples from 24 patients $(92 \%)$ were genetically susceptible to $\mathrm{AG} / \mathrm{CH}$ (in $2 / 3$ cases solo, in $1 / 3$ - in combination with sensitivity to ethambutol (5 samples) and fluoroquinolones (1)). Most samples demonstrated genetic resistance to fluoroquinolones (97\%) and ethambutol (80\%), and $30 \%$ of samples are resistant to aminoglycosides/cyclic peptides.

Among the gyrA mutations, 11 were in codon 90 (A90V), 43 - in codon 94 (of which 4 - D94A, $6-$ D94N, D94Y, $30-$ D94G and $3-$ D94H). No mutation in codon 91 (S91P) was detected. In 22 samples 1 mutation was detected, in $4-2$ mutations, in $6-$ 3 mutations, and in $1-5$ mutations. Among the mutations found in the rrs gene, 8 are in codons 1401-1402 (A1401G, C1402T) and 10 - in codon 1484 (G1484T), all mutations in codons 1401-1402 are combined with the presence of a mutation in codon 1484. Among mutations in $e m b B$ (codon 306) in three cases replacement of M306I = 306 ATG/ATA, M306V, in 26 - replacement of $\mathrm{M} 306 \mathrm{I}=306 \mathrm{ATG} / \mathrm{ATC} / \mathrm{ATT}$ was revealed. In 24 cases only one variant of the mutation is found, in $2-$ both, and in 18 samples in the presence of a mutation there is no marker of wild type.

In conclusion, preliminary data on the genetic structure of MBT strains resistant to rifampicin and second-line anti-tuberculosis drugs were obtained in tuberculosis patients from the Tyumen Region in Siberia. doi: 10.15789/2220-7619-2018-4-6.5

\section{MOLECULAR TYPING OF MYCOBACTERIUM} KANSASII - A GLOBAL PERSPECTIVE

Z. Bakula ${ }^{1}$, F. Arias ${ }^{2}$, E. Bachiyska ${ }^{3}$, E. Borroni ${ }^{4}$, D.M. Cirillo ${ }^{4}$, C. Coulter ${ }^{5}$, C. Giske ${ }^{6}$, J. Humiecka ${ }^{7}$, J. van Ingen ${ }^{8}$, P. Ioannidis ${ }^{9}$, K. Kranzer ${ }^{10}$, U. Kuzmič ${ }^{11}$, K. Levina ${ }^{12}$, T. Lillebæk ${ }^{13}$, I. Mokrousov ${ }^{14}$, K. Morimoto ${ }^{15}$, V. Nikolayevskyy ${ }^{16}$, A. Norman ${ }^{13}$, D. Papaventsis ${ }^{9}$, O. Peuchant ${ }^{17}$, A. Safianowska ${ }^{18}$, V. Ulmann ${ }^{19}$, E. Vasiliauskiene ${ }^{20}$, K. Won-Jung ${ }^{21}$, V. Zhuravlev ${ }^{22}$, M. Žolnir-Dovč ${ }^{11}$, R. Krenke ${ }^{18}$, T. Jagielski ${ }^{1}$

${ }^{I}$ Department of Applied Microbiology, Institute of Microbiology, Faculty of Biology, University of Warsaw, Warsaw, Poland; ${ }^{2}$ Mycobacteria Laboratory, Public Health Institute of Chile, Santiago, Chile; ${ }^{3}$ National Reference Laboratory for Tuberculosis, Sofia, Bulgaria; ${ }^{4}$ Emerging Bacterial Pathogens Unit, San Raffaele Scientific Institute, Milano, Italy; ${ }^{5}$ Queensland Mycobacterial Reference Laboratory, Queensland, Brisbane, Australia; ${ }^{6}$ Department of Clinical Microbiology, Karolinska University Hospital, Solna, Sweden; ${ }^{7}$ Hospital of Infectious Diseases in Warsaw, Warsaw, Poland; ${ }^{8}$ Department of Medical Microbiology, Radboud University Medical Center, Nijmegen, The Netherlands; ${ }^{9}$ National Reference Laboratory for Mycobacteria, "Sotiria" Chest Diseases Hospital of Athens, Greece; ${ }^{10}$ National Reference Center for Mycobacteria, Forschungszentrum Borstel, Leibniz-Zentrum für Medizin und Biowissenschaften, Borstel, Germany; ${ }^{11}$ Laboratory for Mycobacteria, University Clinic of Respiratory and Allergic Diseases Golnik, Golnik, Slovenia; ${ }^{12}$ Mycobacteriology Section Laboratory, North Estonia Medical Centre, Tallinn, Estonia; ${ }^{13}$ International Reference Laboratory of Mycobacteriology, Statens Serum Institut, Copenhagen, Denmark; ${ }^{14}$ Laboratory of Molecular Microbiology, St. Petersburg Pasteur Institute, St. Petersburg, Russia: ${ }^{15}$ Respiratory Disease Center, Fukujuji Hospital, Japan Anti-Tuberculosis Association, Tokyo, Japan; ${ }^{16}$ Imperial College, London, United Kingdom; ${ }^{17}$ Laboratoire de Bactériologie, Centre Hospitalier Universitaire de Bordeaux, Bordeaux, France; ${ }^{18}$ Department of Internal Medicine, Pulmonary Diseases \& Allergy, Warsaw Medical University, Warsaw, Poland; ${ }^{19}$ Institute of Public Health, Ostrava, Czech Republic; ${ }^{20}$ Infectious Diseases and Tuberculosis Hospital, Vilnius University Hospital Santariskiu Klinikos, Lithuania, ${ }^{21}$ Division of Pulmonary and Critical Care Medicine; Department of Medicine, Samsung Medical Center, Sungkyunkwan University School of Medicine, South Korea; ${ }^{22}$ Research Institute of Phthisiopulmonology, St. Petersburg, Russia

To date, over 180 nontuberculous mycobacteria (NTM) species have been identified and almost 30 of these species have been reported as the causative agents of pulmonary and extrapulmonary diseases. Mycobacterium kansasii is the sixth most frequently isolated NTM species across the world. The isolation rate of this pathogen, among other NTM, has been calculated at 5\% in Europe and 4\% globally. In Poland and Slovakia, the recovery of M. kansasii from respiratory samples is particularly high, being $36 \%$ and $35 \%$, respectively.

The genetic heterogeneity of M. kansasii is defined by the presence of seven molecular subtypes. Most of the disease-related strains belong to subtype I and II, while the others (III-VII) have usually been linked to environmental sources. Therefore, subtyping of M. kansasii isolates from human samples may be helpful for clinical diagnosis.

The aim of this study was to determine the distribution of M. kansasii subtypes among clinical isolates from 19 countries on 4 continents.

A total of 475 isolates recovered between 2000 and 2017 from as many patients with suspected $M$. kansasii disease were analyzed. The isolates were collected from 19 coun- 
tries across 4 continents. For PCR restriction-enzyme analysis (PCR-REA) subtyping, protocols described by Telenti et al. (2003) (hsp65 gene) and Bakuła et. al. (2016) (tufgene) were used. The patients were categorized as having M. kansasii disease following the American Thoracic Society 2007 diagnostic criteria.

The vast majority of isolates $(392 ; 82.5 \%)$ presented patterns characteristic for subtype I. Forty-three (9\%) isolates exhibited subtype II pattern. There were $19(4 \%), 2$ $(0.4 \%), 2(0.4 \%)$ and $13(2.7 \%)$ isolates representing subtypes III, IV, V, and VI, respectively. Four $(0.8 \%)$ isolates gave inconsistent results (mixed subtype - I/II). The subtype I-VI isolates were obtained from both disease-associated and non-disease associated cases. Of two subtype IV isolates, one was obtained from a non-disease associated case, and the other one from a patient with unknown status. For two subtype $\mathrm{V}$ isolates, data concerning M. kansasii disease were unavailable.

The highest frequency of $M$. kansasii subtype I isolations was observed for Poland $(140 / 142 ; 98.6 \%)$, and the lowest for Estonia $(2 / 7 ; 28.6 \%)$.

This study demonstrated that subtype I represented the vast majority of $M$. kansasii clinical isolates worldwide. Since all M. kansasii subtypes detected (I-VI) were isolated from both disease-related and non-related cases, subtyping of the species does not permit differentiation between disease and non-disease states that did and did not cause definite disease. Furthermore, the genetic diversity of the M. kansasii population showed important regional variations.

The study was performed within the framework of the Fight Against Tuberculosis in Central \& Eastern Europe consortium (https://fate-consortium.org).

The study was supported by the National Centre for Research and Development "LIDER" Programme (LIDER/044/457/L-4/12/ NCBR/2013) and the Faculty of Biology "DSM" grant (501D114-86-0115000-01).

\section{6}

doi: 10.15789/2220-7619-2018-4-6.6

\section{GENETIC DIVERSITY OF MULTIDRUG-RESISTANT MYCOBACTERIUM TUBERCULOSIS ISOLATES IN PAKISTAN}

Z. Bakuła ${ }^{1}$, M. Pleń ${ }^{1}$, H. Javed ${ }^{2}$, H.J. Hashmi ${ }^{2}$, Z. Tahir ${ }^{3}$, K. Roeske ${ }^{1}$, N. Jamil ${ }^{2}$, T. Jagielski ${ }^{3}$

${ }^{1}$ Department of Applied Microbiology, Institute of Microbiology, Faculty of Biology, University of Warsaw, Poland; ${ }^{2}$ Department of Microbiology and Molecular Genetics, University of the Punjab, Lahore, Pakistan; ${ }^{3}$ Provincial TB Control Program, Lahore, Pakistan

Tuberculosis $(\mathrm{TB})$ remains an inglorious leader among infectious diseases in mortality, with its annual toll of 1.7 million lives worldwide. Pakistan ranks $5^{\text {th }}$ among the world's highest TB burden countries and the $6^{\text {th }}$ among countries with the highest burden of drug-resistant TB, including multi-drug resistant (MDR)-TB. However, very limited data are available on the genetic structure of $M$. $t u$ berculosis strains circulating in this country.

The objective of this study was to explore the genetic diversity of multidrug-resistant $M$. tuberculosis isolates from Pakistan with two different methodologies, i.e. spoligotyping and 24-loci MIRU-VNTR typing.

The study included 130 MDR-TB isolates, recovered from as many patients from Pakistan, between January 2013 and June 2015. Conventional drug susceptibility testing was performed using the standard $1 \%$ proportion method on the Löwenstein-Jensen medium, as described elsewhere. Spoligotyping was performed with a commer- cially available kit (Mapmygenome India Ltd., Madhapur, India) according to the manufacturer's protocol. MIRUVNTR analysis was carried out at 24 loci, as described earlier. Phylogenetic clades of M. tuberculosis were assigned according to signatures provided in the SITVIT database (http://www.pasteur-guadeloupe.fr:8081/SITVIT_ ONLINE).

Spoligotypes were obtained for $127(97.8 \%)$ isolates. Based on a SIT number in the SITVIT database, all isolates presented 53 different profiles split into 14 clusters $(\mathrm{n}=88,69.3 \%, 2-30$ isolates per cluster) and $39(30.7 \%)$ unique patterns. MIRU-VNTR typing identified 128 unique types $(98.5 \%)$ and one cluster $(\mathrm{n}=2,1.5 \%)$. When spoligotyping and MIRU-VNTR typing was used in combination, only two, out of 130 isolates, clustered both in both methods, resulting in a clustering rate of $1.5 \%$.

Upon phylogenetic analysis, 101 (77.7\%) isolates were classified into 12 clades, with the most prevalent being CAS1_DELHI $(\mathrm{n}=53,41.7 \%)$ followed by T1 $(\mathrm{n}=$ $14,11 \%)$ and BEIJING $(\mathrm{n}=10,7.8 \%)$. The remaining 9 families (CAS, MANU2, EAI5, T2, LAM10_CAM, $\mathrm{H} 1, \mathrm{X} 1, \mathrm{H} 4$ and CAS2) involved $24(18.9 \%)$ isolates. Twenty-six (20.5\%) isolates could not be assigned to any specific lineage.

This study provides a snapshot of the genetic diversity of M. tuberculosis strains circulating in Pakistan. The compactness of the drug resistant $M$. tuberculosis population structure was apparent, as three major lineages, i.e. CAS1_DELHI, T1, and BEIJING comprised more than half $(\overline{60} .6 \%)$ of the isolates studied. Furthermore, the exceptionally low clustering rate

Suggest that recent transmission does not play an important role in the incidence of MDR-TB in Pakistan.

6.7 doi: $10.15789 / 2220-7619-2018-4-6.7$

\section{UPDATE ON VIRULENCE FACTORS IN MYCOBACTERIA}

\section{R. Brosch}

\section{Institut Pasteur, Paris, France}

Although the majority of mycobacteria represent harmless environmental bacteria, a few mycobacterial species have evolved into major human pathogens. Mycobacterium tuberculosis, the etiological agent of human tuberculosis, is the most dominant mycobacterial pathogen in terms of global patient numbers and gravity of disease.

The molecular mechanisms by which $M$. tuberculosis induces disease are complex and result from a long-lasting host-pathogen co-evolution that might have started already by its Mycobacterium canettii-like progenitors. Recent research has revealed numerous factors implicated in the pathogenesis of M. tuberculosis, although the pathogen still holds many secrets of its successful strategy to circumvent host defences and persist in the host. As many pathogenicity factors relate to the exchange and secretion of biomolecules by $M$. tuberculosis, special emphasis is given to secretion pathways that enable $M$. tuberculosis to circumvent immune defence mechanisms mounted by the host. These factors might represent new, alternative targets for development of combination therapies that would enhance the efficacy of the immune system in controlling $M$. tuberculosis infections. Similarly, selected secretion systems may also represent important virulence factors in selected non-tuberculous mycobacteria. Here, recent insights into evolution of selected factors of M. tuberculosis and selected other mycobacteria that are involved in host-pathogen interaction will be discussed. 